Piero D’Ancona · Damiano Foschi · Sigmund Selberg

\title{
Null structure and almost optimal local regularity for the Dirac-Klein-Gordon system
}

Received May 12, 2006 and in revised form August 7, 2006

\begin{abstract}
We prove almost optimal local well-posedness for the coupled Dirac-Klein-Gordon (DKG) system of equations in $1+3$ dimensions. The proof relies on the null structure of the system, combined with bilinear spacetime estimates of Klainerman-Machedon type. It has been known for some time that the Klein-Gordon part of the system has a null structure; here we uncover an additional null structure in the Dirac equation, which cannot be seen directly, but appears after a duality argument.
\end{abstract}

Keywords. Dirac equation, Klein-Gordon equation, null structure, local well-posedness, null form estimates

\section{Introduction}

In standard notation, the coupled Dirac-Klein-Gordon (DKG) system of equations on $\mathbb{R}^{1+3}$ reads

$$
\begin{cases}\left(-i \gamma^{\mu} \partial_{\mu}+M\right) \psi=\phi \psi & (M \geq 0), \\ \left(-\square+m^{2}\right) \phi=\psi^{\dagger} \gamma^{0} \psi & \left(\square=-\partial_{t}^{2}+\Delta, m \geq 0\right),\end{cases}
$$

where the unknowns are (i) a spinor field $\psi(t, x) \in \mathbb{C}^{4}$, regarded as a column vector in $\mathbb{C}^{4}$, and (ii) a real scalar field $\phi(t, x)$. We use coordinates $t=x^{0}, x=\left(x^{1}, x^{2}, x^{3}\right)$ on $\mathbb{R}^{1+3}$, and write $\partial_{\mu}=\partial / \partial x^{\mu}$. Greek indices $\mu, v$ etc. range over $0,1,2,3$, Roman indices $j, k$ etc. over $1,2,3$, and repeated indices are summed over these ranges. Thus, $\gamma^{\mu} \partial_{\mu}=\sum_{\mu=0}^{3} \gamma^{\mu} \partial_{\mu}$, where $\left\{\gamma^{\mu}\right\}_{\mu=0}^{3}$ are the $4 \times 4$ Dirac matrices, given in $2 \times 2$ block form by

$$
\gamma^{0}=\left(\begin{array}{cc}
I & 0 \\
0 & -I
\end{array}\right), \quad \gamma^{j}=\left(\begin{array}{cc}
0 & \sigma^{j} \\
-\sigma^{j} & 0
\end{array}\right)
$$

P. D’Ancona: Dipartimento di Matematica, Università di Roma "La Sapienza", Piazzale Aldo Moro 2, I-00185 Rome, Italy; e-mail: dancona@mat.uniroma1.it

D. Foschi: Dipartimento di Matematica, Università di Ferrara, via Macchiavelli 35, I-44100 Ferrara, Italy; e-mail: damiano.foschi@unife.it

S. Selberg: Department of Mathematical Sciences, Norwegian University of Science and Technology, Alfred Getz' vei 1, N-7491 Trondheim, Norway; e-mail: sigmund.selberg@ math.ntnu.no

Mathematics Subject Classification (2000): 35Q40, 35L70 
where

$$
\sigma^{1}=\left(\begin{array}{ll}
0 & 1 \\
1 & 0
\end{array}\right), \quad \sigma^{2}=\left(\begin{array}{cc}
0 & -i \\
i & 0
\end{array}\right), \quad \sigma^{3}=\left(\begin{array}{cc}
1 & 0 \\
0 & -1
\end{array}\right)
$$

are the Pauli matrices. $\psi^{\dagger}$ denotes the adjoint, i.e., the conjugate transpose, hence

$$
\psi^{\dagger} \gamma^{0} \psi \equiv\left|\psi_{1}\right|^{2}+\left|\psi_{2}\right|^{2}-\left|\psi_{3}\right|^{2}-\left|\psi_{4}\right|^{2},
$$

where $\psi_{1}, \ldots, \psi_{4}$ are the components of $\psi$. The following related matrices occur frequently below:

$$
\beta \equiv \gamma^{0}, \quad \alpha^{j} \equiv \gamma^{0} \gamma^{j}=\left(\begin{array}{cc}
0 & \sigma^{j} \\
\sigma^{j} & 0
\end{array}\right), \quad S^{m} \equiv i \gamma^{k} \gamma^{l}=\left(\begin{array}{cc}
\sigma^{m} & 0 \\
0 & \sigma^{m}
\end{array}\right)
$$

where $(k, l, m)$ is any cyclic permutation of $(1,2,3)$. Denoting by $\delta^{j k}$ the Kronecker delta and by $\varepsilon^{j k l}$ the completely antisymmetric tensor 1 we have the identities

$$
\begin{aligned}
\alpha^{j} \beta & =-\beta \alpha^{j}, \\
\alpha^{j} \alpha^{k} & =-\alpha^{k} \alpha^{j}+2 \delta^{j k} I, \\
\alpha^{j} \alpha^{k} & =\delta^{j k} I+i \epsilon^{j k l} S^{l} .
\end{aligned}
$$

Also, $\beta^{2}=\left(\alpha^{j}\right)^{2}=I$ and $\beta^{\dagger}=\beta,\left(\alpha^{j}\right)^{\dagger}=\alpha^{j}$.

Concerning the Cauchy problem, the most fundamental question is whether global regularity holds, i.e., given smooth, compactly supported initial data, does DKG have a smooth solution for all times $t>0$ ? For small data, the answer is yes (see [2, 20, 11]), but for large data it remains an open question, except in the $1+1$ dimensional case (see Chadam [9]). In 1+3 dimensions, global regularity is known only for a very special class of (large) data: Chadam and Glassey [10] proved it for data satisfying the constraints $\psi_{1}(0, x)=\bar{\psi}_{4}(0, x)$ and $\psi_{2}(0, x)=-\bar{\psi}_{3}(0, x)$, which imply that $\psi^{\dagger} \gamma^{0} \psi$ vanishes initially, and in fact stays zero in the evolution; later, Bachelot [3] extended this result to cover also small perturbations around such data. Another global result is proved in [12] for data with special symmetry properties.

In order to make progress on the global regularity question, a natural strategy is to study local (in time) well-posedness (LWP) for low regularity data, and then try to exploit the conserved quantities of the system. This strategy was successfully implemented for the Maxwell-Klein-Gordon (MKG) and Yang-Mills (YM) equations by Klainerman and Machedon [23, 24], who proved LWP for data with finite energy and then used the conservation of energy to push this to a global result, thus recovering, in particular, the classical result of Eardley and Moncrief [13]. Compared to MKG and YM, however, DKG has the unpleasant feature that the conserved energy $\int e(\phi, \psi) d x=$ const has a density which is not positive definite (see [18]):

$$
e(\phi, \psi)=\operatorname{Im}\left(\psi^{\dagger} \alpha^{j} \partial_{j} \psi\right)-(M-g \phi) \psi^{\dagger} \beta \psi-\frac{1}{2}\left(\left(\partial_{t} \phi\right)^{2}+|\nabla \phi|^{2}+m^{2} \phi^{2}\right) .
$$

1 So $\varepsilon^{123}=\varepsilon^{231}=\varepsilon^{312}=-\varepsilon^{213}=-\varepsilon^{321}=-\varepsilon^{132}=1$, and all other $\varepsilon^{j k l}=0$. 
On the other hand, one does have the conservation of charge:

$$
\int|\psi(t, x)|^{2} d x=\text { const }
$$

which was a key ingredient in Chadam's proof of global regularity in the $1+1$ dimensional case [9] (see also [7, 14]).

We are interested in LWP of the Cauchy problem with data

$$
\psi(0, x)=\psi_{0}(x), \quad \phi(0, x)=\phi_{0}(x), \quad \partial_{t} \phi(0, x)=\phi_{1}(x)
$$

with regularity $\left(\psi_{0}, \phi_{0}, \phi_{1}\right) \in H^{s} \times H^{r} \times H^{r-1}$ for minimal $s, r \in \mathbb{R}$. Here $H^{s}=H^{s}\left(\mathbb{R}^{3}\right)$ is the Sobolev space with norm

$$
\|f\|_{H^{s}}=\left\|\langle\xi\rangle^{s} \widehat{f}(\xi)\right\|_{L_{\xi}^{2}}
$$

where $\widehat{f}(\xi)$ denotes the Fourier transform of $f(x)$ and $\langle\cdot\rangle=1+|\cdot|$. We denote by $\dot{H}^{s}$ the corresponding homogeneous space, with norm $\|f\|_{\dot{H}^{s}}=\left\||\xi|^{s} \widehat{f}(\xi)\right\|_{L_{\xi}^{2}}$.

To get an idea of the minimal regularity required for LWP, one can apply the usual scaling heuristic. In the massless case $M=m=0$, DKG is invariant under the rescaling

$$
\psi(t, x) \mapsto \frac{1}{L^{3 / 2}} \psi\left(\frac{t}{L}, \frac{x}{L}\right), \quad \phi(t, x) \mapsto \frac{1}{L} \phi\left(\frac{t}{L}, \frac{x}{L}\right),
$$

hence the scale invariant data space is (in $1+3$ dimensions)

$$
\left(\psi_{0}, \phi_{0}, \phi_{1}\right) \in L^{2} \times \dot{H}^{1 / 2} \times \dot{H}^{-1 / 2},
$$

and one does not expect well-posedness below this regularity. The scaling also suggests that $r=1 / 2+s$ is the natural choice.

On the other hand, DKG is a system of nonlinear wave equations with quadratic nonlinearities (as can be seen by squaring the Dirac equation), and for such equations it is well known (see [28]) that, due to nonlinear effects, one cannot hope to reach the regularity predicted by scaling unless Klainerman's null condition is satisfied. The null condition is a condition on the symbol of the quadratic nonlinearities, which cancels the most dangerous interactions in a product of free waves.

The local well-posedness results for DKG are listed in Table 1 . By classical methods (energy estimates and Sobolev embeddings) one can prove LWP for data $\left(\psi_{0}, \phi_{0}, \phi_{1}\right) \in$ $H^{1+\varepsilon} \times H^{3 / 2+\varepsilon} \times H^{1 / 2+\varepsilon}$ for any $\varepsilon>0$. Bachelot [1] proved that the $\varepsilon$ can be removed. By using Strichartz type estimates for the homogeneous wave equation one can prove local well-posedness in $H^{1 / 2+\varepsilon} \times H^{1+\varepsilon} \times H^{\varepsilon}$ (see [32, 6]), but in order to lower the regularity further one needs null structure. Klainerman and Machedon [21] demonstrated a null structure, via an auxiliary variable, in the quadratic form $\psi^{\dagger} \gamma^{0} \psi$ appearing in the Klein-Gordon equation. Later, Beals and Bezard [4] found a more direct expression of this null structure by using the eigenspace projections of the Dirac operator (thus avoiding the auxiliary variable), and applied it to gain some spacetime regularity (see Table 1 ). 
Table 1. Local well-posedness results for DKG

\begin{tabular}{|ll|c|c|}
\hline & & $\left(\psi_{0}, \phi_{0}\right) \in$ & $(\psi, \phi) \in$ \\
\hline & classical methods & $H^{1+\varepsilon} \times H^{3 / 2+\varepsilon}$ & $C\left([0, T] ; H^{1+\varepsilon}\right) \times C\left([0, T] ; H^{3 / 2+\varepsilon}\right)$ \\
\hline 1984 & Bachelot [1] & $H^{1} \times H^{3 / 2}$ & $C\left([0, T] ; H^{1}\right) \times C\left([0, T] ; H^{3 / 2}\right)$ \\
\hline 1993 & Strichartz est. [32, 6] & $H^{1 / 2+\varepsilon} \times H^{1+\varepsilon}$ & $C\left([0, T] ; H^{1 / 2+\varepsilon}\right) \times C\left([0, T] ; H^{1+\varepsilon}\right)$ \\
\hline 1996 & Beals-Bezard [4] & $H^{1} \times H^{2}$ & $H^{1}\left([0, T] \times \mathbb{R}^{3}\right) \times H^{2}\left([0, T] \times \mathbb{R}^{3}\right)$ \\
\hline 1999 & Bournaveas [6] & $H^{1 / 2} \times H^{1}$ & $C\left([0, T] ; H^{1 / 2}\right) \times C\left([0, T] ; H^{1}\right)$ \\
\hline 2004 & Gibbeson [17] & $H^{4 / 9} \times H^{8 / 9}$ & $C\left([0, T] ; H^{4 / 9}\right) \times C\left([0, T] ; H^{8 / 9}\right)$ \\
\hline 2005 & Fang-Grillakis [15] & $H^{1 / 4+\varepsilon} \times H^{1}$ & $H^{1 / 4+\varepsilon, 1 / 2+\varepsilon} \times H^{1,1 / 2+\varepsilon}$ \\
\hline 2006 & D. - F. - S. & $H^{\varepsilon} \times H^{1 / 2+\varepsilon}$ & $X^{\varepsilon, 1 / 2+\varepsilon} \times H^{1 / 2+\varepsilon, 1 / 2+\varepsilon}$ \\
\hline & scaling & $L^{2} \times \dot{H}^{1 / 2}$ & \\
\hline
\end{tabular}

On the other hand, Bournaveas [6], following the idea of Klainerman and Machedon [21], found a null structure in the Dirac part of the system, and used this to get rid of the epsilon in the $H^{1 / 2+\varepsilon} \times H^{1+\varepsilon} \times H^{\varepsilon}$ result, i.e., he proved LWP in the "energy class" $H^{1 / 2} \times H^{1} \times L^{2}$; this was improved slightly in the $\mathrm{PhD}$ thesis of Gibbeson [17]. While the null structure found by Bournaveas helps to a certain extent, it has the drawback that it involves squaring the Dirac equation, which creates serious difficulties at very low regularity. It should be noted, however, that $X^{s, b}$ type spaces were not used in [6] (although they are lurking in the background in Lemma 2 of the same paper), which allow one to take maximum advantage of the null structure. Using the machinery of these spaces together with the null structure proved in [4, 6] and bilinear spacetime estimates of Klainerman-Machedon type, one can, not surprisingly, improve the result from [6]. In fact, quite recently Fang and Grillakis [15] have proved LWP in $H^{s} \times H^{1} \times L^{2}$ for all $1 / 4<s \leq 1 / 2$.

The new idea which drives the present paper is that the null form $\psi^{\dagger} \gamma^{0} \psi$ occurs not only in the Klein-Gordon part, but in fact also in the Dirac part of the system, as can be seen via a duality argument. The resulting structure dramatically simplifies the analysis of the system, and allows us, using some fairly standard bilinear spacetime estimates of Klainerman-Machedon type, to set up a contraction in $X^{s, b}$ type spaces and prove local well-posedness arbitrarily close to the scale invariant regularity:

Theorem 1. DKG in $1+3$ dimensions is LWP for data

$$
\left(\psi_{0}, \phi_{0}, \phi_{1}\right) \in H^{\varepsilon} \times H^{1 / 2+\varepsilon} \times H^{-1 / 2+\varepsilon}
$$

for all $\varepsilon>0$.

The structure uncovered here for the DKG system can be seen as an analogue of that found by Machedon and Sterbenz [29] for the Maxwell-Klein-Gordon system in Coulomb gauge. The systems are somewhat related, in that both can be seen as simplifications of the 
Maxwell-Dirac system, but Maxwell-Klein-Gordon does not have the spinor structure which is at the heart of our analysis of DKG.

Note that Theorem 1 leaves open the critical case $(\varepsilon=0)$. It is reasonable to expect that DKG is globally well posed for small data in some Besov norm with the same scaling as $L^{2} \times \dot{H}^{1 / 2} \times \dot{H}^{-1 / 2}$, but we do not consider this question here.

Since this work was completed, our method has been applied by Pecher [31] to treat the one-dimensional DKG system, which has also been studied independently by Machihara [30].

\section{Preliminaries}

For convenience we rewrite (1.1) in a slightly different form, multiplying the Dirac equation on the left by $\beta=\gamma^{0}$ to get

$$
\left\{\begin{array}{l}
-i\left(\partial_{t}+\alpha \cdot \nabla\right) \psi=-M \beta \psi+\phi \beta \psi, \\
\square \phi=m^{2} \phi-\langle\beta \psi, \psi\rangle_{\mathbb{C}^{4}} .
\end{array}\right.
$$

Here $\alpha$ denotes the vector $\left(\alpha^{1}, \alpha^{2}, \alpha^{3}\right)$ whose components are the Dirac matrices $\alpha^{j}=$ $\gamma^{0} \gamma^{j}$; thus, $\alpha \cdot \nabla=\alpha^{j} \partial_{j}$. Further, $\langle\cdot, \cdot\rangle_{\mathbb{C}^{4}}$ denotes the standard inner product on $\mathbb{C}^{4}$.

The operator $-i\left(\partial_{t}+\alpha \cdot \nabla\right)$ is rather complicated, since $-i \alpha \cdot \nabla$ mixes the components of the spinor it acts on. To simplify matters, it is natural to diagonalize by decomposing the spinor field relative to an eigenbasis of the operator $-i \alpha \cdot \nabla$. The symbol of the latter is $\alpha \cdot \xi\left(\xi \in \mathbb{R}^{3}\right)$. A quick calculation using $\left[1.3\right.$ gives $(\alpha \cdot \xi)^{2}=|\xi|^{2} I$, hence the eigenvalues of $\alpha \cdot \xi$ are $\pm|\xi|$. By symmetry, each eigenspace is two-dimensional, and the projections onto these eigenspaces are given by

$$
\Pi_{ \pm}(\xi)=\frac{1}{2}(I \pm \hat{\xi} \cdot \alpha) \quad \text { where } \quad \hat{\xi} \equiv \frac{\xi}{|\xi|}
$$

Now write

$$
\psi=\psi_{+}+\psi_{-} \quad \text { where } \quad \psi_{ \pm}=\Pi_{ \pm}(D) \psi
$$

Here $D=\nabla / i$, which has Fourier symbol $\xi$. Throughout we use the notation $h(D)$ for the multiplier with symbol $h(\xi)$, for a given function $h: \mathbb{R}^{3} \rightarrow \mathbb{C}$.

Applying $\Pi_{ \pm}(D)$ to the Dirac equation in 2.1, and using the identities

$$
-i \alpha \cdot \nabla=|D| \Pi_{+}(D)-|D| \Pi_{-}(D),
$$

and

$$
\Pi_{ \pm}(\xi) \beta=\beta \Pi_{\mp}(\xi)
$$

(the latter due to $(1.2)$ ), we obtain

$$
\left\{\begin{array}{l}
\left(-i \partial_{t}+|D|\right) \psi_{+}=-M \beta \psi_{-}+\Pi_{+}(D)(\phi \beta \psi), \\
\left(-i \partial_{t}-|D|\right) \psi_{-}=-M \beta \psi_{+}+\Pi_{-}(D)(\phi \beta \psi), \\
\square \phi=m^{2} \phi-\langle\beta \psi, \psi\rangle_{\mathbb{C}^{4}}
\end{array}\right.
$$

which is the system we shall work with. 
We iterate $\psi_{ \pm}$and $\phi$ in $X^{s, b}$ type spaces associated to the operators $-i \partial_{t} \pm|D|$ and $\square$, whose symbols are $\tau \pm|\xi|$ and $\tau^{2}-|\xi|^{2}$, respectively. The notation $\widetilde{u}(\tau, \xi)$ is used for the spacetime Fourier transform of a function $u(t, x)$.

Definition 1. Let $X_{ \pm}^{s, b}(s, b \in \mathbb{R})$ be the completion of the Schwartz space $\mathcal{S}\left(\mathbb{R}^{1+3}\right)$ with respect to the norm

$$
\|u\|_{X_{ \pm}^{s, b}}=\left\|\langle\xi\rangle^{s}\langle\tau \pm|\xi|\rangle^{b} \widetilde{u}(\tau, \xi)\right\|_{L_{\tau, \xi}^{2}}
$$

where as before $\langle\cdot\rangle=1+|\cdot|$. Note that $\|u\|_{X_{ \pm}^{s, b}}=\left\|\langle D\rangle^{s}\left\langle-i \partial_{t} \pm|D|\right\rangle^{b} u\right\|_{L_{t, x}^{2}}$, by Plancherel's theorem.

Spaces of this type were first used by Bourgain [5] for periodic solutions of nonlinear Schrödinger and KdV equations, and later by Kenig, Ponce and Vega [19] in the nonperiodic case. Similar spaces for the wave equation were first used by Klainerman and Machedon [25], who used the notation $H^{s, b}$. Here we rely on a slight variation of the $H^{s, b}$ spaces of Klainerman and Machedon, introduced in [33] (alternatively, see [34]) and applied in [27], where they are referred to as wave-Sobolev spaces. To describe these spaces, it is convenient to introduce mulipliers $D_{ \pm}$with symbols $|\tau| \pm|\xi|$. Thus, $\square=D_{+} D_{-}$.

Definition 2. Let $H^{s, b}$ and $\mathcal{H}^{s, b}(s, b \in \mathbb{R})$ be the completions of $\mathcal{S}\left(\mathbb{R}^{1+3}\right)$ with respect to the norms

$$
\begin{aligned}
\|u\|_{H^{s, b}} & =\left\|\langle D\rangle^{s}\left\langle D_{-}\right\rangle^{b} u\right\|_{L_{t, x}^{2}}=\left\|\langle\xi\rangle^{s}\langle|\tau|-|\xi|\rangle^{b} \widetilde{u}(\tau, \xi)\right\|_{L_{(\tau, \xi)}^{2}}, \\
\|u\|_{\mathcal{H}^{s, b}} & =\|u\|_{H^{s, b}}+\left\|\partial_{t} u\right\|_{H^{s-1, b}},
\end{aligned}
$$

respectively. Observe that the last norm is equivalent to $\left\|\langle D\rangle^{s-1}\left\langle D_{+}\right\rangle\left\langle D_{-}\right\rangle^{b} u\right\|_{L_{t, x}^{2}}$.

In Section 4 we recall the basic facts concerning these spaces, for the convenience of the reader. For more details and applications, the reader may want to look at [27, 36].

We shall also need the restrictions of the above spaces to a time slab

$$
S_{T}=(0, T) \times \mathbb{R}^{3},
$$

since we study local in time solutions. The restriction $X_{ \pm}^{s, b}\left(S_{T}\right)$ is a Banach space with respect to the norm

$$
\|u\|_{X_{ \pm}^{s, b}\left(S_{T}\right)}=\inf \left\{\|v\|_{X_{ \pm}^{s, b}}: v \in X_{ \pm}^{s, b} \text { and } v=u \text { on } S_{T}\right\}
$$

In fact, the completeness follows from a basic result of abstract functional analysis, since $X_{ \pm}^{s, b}\left(S_{T}\right)$ is nothing else than the quotient space $X_{ \pm}^{s, b} / \mathcal{M}_{ \pm}$, where $\mathcal{M}_{ \pm}$is the closed subspace $\left\{v \in X_{ \pm}^{s, b}: v=0\right.$ on $\left.S_{T}\right\}$. The restriction spaces $H^{s, b}\left(S_{T}\right)$ and $\mathcal{H}^{s, b}\left(S_{T}\right)$ are defined analogously. 


\section{Null structure}

In this section we discuss the null structure in DKG. First, however, let us recall the null condition of Klainerman and give a heuristic argument showing its significance for regularity of nonlinear waves. To this end, consider a nonlinear wave equation with a quadratic nonlinearity, $\square u=B(u, u)$, where $B$ is a bilinear operator given by a Fourier symbol $b$. Specifically, if $X=(\tau, \xi), Y=(\lambda, \eta)$ and $Z=(\mu, \zeta)$ are vectors in Fourier space $\mathbb{R} \times \mathbb{R}^{3}, B$ is of the form

$$
\begin{aligned}
{[B(v, v)]^{\sim}(X) } & =\iint_{Y+Z=X} b(Y, Z) \widetilde{v}(Y) \widetilde{v}(Z) d Y d Z \\
& =\int b(Y, X-Y) \widetilde{v}(Y) \widetilde{v}(X-Y) d Y
\end{aligned}
$$

We say $X=(\tau, \xi)$ is null if it lies on the null cone $|\tau|=|\xi|$; this is equivalent to saying that the symbol $\square(X) \equiv \tau^{2}-|\xi|^{2}$ of the wave operator vanishes on $X$. Let us suppose $v$ is a free wave, $\square v=0$, so that $\widetilde{v}$ is a measure supported on the null cone, and let us look at the regularity of $u$ solving $\square u=B(v, v)$. (This problem arises naturally when solving the nonlinear problem by iteration.) In Fourier space,

$$
\square(X) \widetilde{u}(X)=[B(v, v)]^{\sim}(X),
$$

so one gains a lot of regularity when $X$ is away from the null cone $(|\square(X)| \gtrsim 1)$. Near the cone, things are not so favorable, but if it happens that $[B(v, v)]^{\sim}(X)$ vanishes (to some order) when $X$ is null, this should improve the regularity in this difficult region. But $X=Y+Z$, where $Y, Z$ are null (since now $v$ in (3.1) is a free wave), hence $X$ is null if and only if $Y, Z$ are parallel. One concludes that $[B(v, v)]^{\sim}(X)$ vanishes for null $X$ if Klainerman's null condition is satisfied, i.e., if

$$
b(Y, Z)=0 \quad \text { for } Y, Z \text { null parallel. }
$$

Remark 1. Note that if two null vectors $Y=(\lambda, \eta)$ and $Z=(\mu, \zeta)$ are on the same component of the cone, i.e., if $\lambda$ and $\mu$ have the same sign, then they are parallel if and only if $\angle(\eta, \zeta)=0$, whereas if they are on opposite components of the cone, the condition is $\angle(\eta,-\zeta)=0$. Here and throughout we use the notation $\angle(\eta, \zeta)$ for the angle between two vectors in $\mathbb{R}^{3}$.

Let us now turn to the null structure in DKG, starting with the Klein-Gordon part of the system:

$$
\square \phi=-\langle\beta \psi, \psi\rangle_{\mathbb{C}^{4}}
$$

For simplicity we set $M=m=0$ in this section.

Since we deal with spinors, the formulation of the null condition differs somewhat from the above. First observe that

$$
\left[\langle\beta \psi, \psi\rangle_{\mathbb{C}^{4}}\right]^{\sim}(X)=\iint_{Y+Z=X}\langle\beta \widetilde{\psi}(Y), \widetilde{\psi}(-Z)\rangle_{\mathbb{C}^{4}} d Y d Z,
$$


where the minus sign in front of $Z$ stems from the complex conjugation in the inner product. Second, 3.2 was derived from the action of (3.1) on a free wave $\square v=0$, whereas in our case there are two separate species of free waves, namely $\psi_{ \pm}$satisfying $\left(-i \partial_{t} \pm|D|\right) \psi_{ \pm}=0$ (cf. 2.4)). Taking data $\psi(0, x)=\psi_{0}(x)$ we have

$$
\psi_{ \pm}(t)=e^{\mp i t|D|} \psi_{0}^{ \pm} \quad \text { where } \quad \psi_{0}^{ \pm}=\Pi_{ \pm}(D) \psi_{0} .
$$

The spacetime Fourier transforms

$$
\widetilde{\psi}_{ \pm}(Y)=\delta(\lambda \pm|\eta|) \widehat{\psi}_{0}^{ \pm}(\eta) \quad(Y=(\lambda, \eta))
$$

are supported on opposite components $-\lambda= \pm|\eta|$ of the null cone $|\lambda|=|\eta|$. Using this information we can state the null condition for $\langle\beta \psi, \psi\rangle_{\mathbb{C}^{4}}$ with $\psi$ replaced by $\psi_{ \pm}$, for all possible combinations of signs (as before, $Y=(\lambda, \eta)$ and $Z=(\mu, \zeta)$ ):

(N1) In the ++ and -- cases, i.e., taking $\left\langle\beta \psi_{+}, \psi_{+}\right\rangle_{\mathbb{C}^{4}}$ or $\left\langle\beta \psi_{-}, \psi_{-}\right\rangle_{\mathbb{C}^{4}}$ in 3.4), we see that $Y, Z$ are on opposite components of the cone (because $Y,-Z$ evidently are on the same component), hence the null condition (cf. Remark 1) says that the (matrix-valued) symbol should vanish when $\angle(\eta,-\zeta)=0$.

(N2) In the +- and -+ cases, $Y, Z$ are on the same component of the cone, hence the null condition says that the symbol should vanish when $\angle(\eta, \zeta)=0$.

This null condition is indeed satisfied by the symbol of

$$
\left(\psi, \psi^{\prime}\right) \mapsto\left\langle\beta \Pi_{+}(D) \psi, \Pi_{ \pm}(D) \psi^{\prime}\right\rangle_{\mathbb{C}^{4}}
$$

This was proved already in [21, 4], but we give a considerably simpler proof below. The main new contribution in the present paper, however, is the fact that the null bilinear forms (3.7) occur not only in the Klein-Gordon part of the system, but also in the Dirac part. To see this requires a duality argument which we now outline. To show the main idea unobscured by technical issues, we prefer to present first a heuristic argument corresponding to the critical regularity $\psi_{ \pm} \in X_{ \pm}^{0,1 / 2}$; the rigorous proof of Theorem 1 is then given in the following sections.

In the following heuristic we take zero initial data for $\phi$, so that $\phi=-\square^{-1}\langle\beta \psi, \psi\rangle$, where $\square^{-1} F$ denotes the solution of $\square u=F$ with vanishing initial data. The Dirac part of the system (2.4) then reads (from now on we drop the index $\mathbb{C}^{4}$ on the inner product)

$$
\left(-i \partial_{t} \pm|D|\right) \psi_{ \pm}=\Pi_{ \pm}(D) F \quad \text { where } \quad F=\left(-\square^{-1}\langle\beta \psi, \psi\rangle\right) \beta \psi
$$

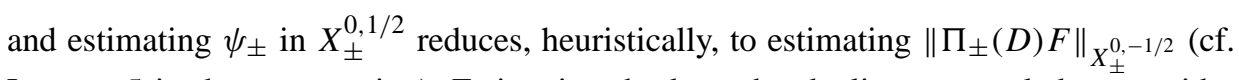
Lemma 5 in the next section). Estimating the latter by duality, we are led to consider integrals, for spinor-valued $\psi^{\prime} \in X_{ \pm}^{0,1 / 2}$

$$
\begin{aligned}
\iint\left\langle\Pi_{ \pm}(D) F, \psi^{\prime}\right\rangle d t d x & =\iint\left\langle F, \Pi_{ \pm}(D) \psi^{\prime}\right\rangle d t d x \\
& =-\iint\left(\square^{-1}\langle\beta \psi, \psi\rangle\right)\left\langle\beta \psi, \Pi_{ \pm}(D) \psi^{\prime}\right\rangle d t d x
\end{aligned}
$$

so indeed, 3.7 crops up one more time. 
In fact, the complete null structure of DKG can be elegantly summed up in a single line: Splitting the fields in the last integral using $\Pi_{ \pm}(D)$, we end up with

$$
\iint\left(\square^{-1}\left\langle\beta \Pi_{ \pm}(D) \psi, \Pi_{ \pm}(D) \psi\right\rangle\right) \cdot\left\langle\beta \Pi_{ \pm}(D) \psi, \Pi_{ \pm}(D) \psi^{\prime}\right\rangle d t d x
$$

for all possible combinations of signs. Replacing $\square^{-1}$ by $|\square|^{-1}$ and distributing it equally over the two factors (this particular heuristic is based on Plancherel's theorem) yields

$$
\iint\left(|\square|^{-1 / 2}\left\langle\beta \Pi_{ \pm}(D) \psi, \Pi_{ \pm}(D) \psi\right\rangle\right) \cdot\left(|\square|^{-1 / 2}\left\langle\beta \Pi_{ \pm}(D) \psi, \Pi_{ \pm}(D) \psi^{\prime}\right\rangle\right) d t d x
$$

The last integral embodies the complete null structure in DKG, and shows the striking symmetry of the system. It suggests that the key problem is to prove the bilinear "estimate"

$$
\left\||\square|^{-1 / 2}\left\langle\beta \Pi_{+}(D) \psi, \Pi_{ \pm}(D) \psi^{\prime}\right\rangle\right\|_{L_{t, x}^{2}} \lesssim\|\psi\|_{X_{+}^{0,1 / 2} \|}\left\|\psi^{\prime}\right\|_{X_{ \pm}^{0,1 / 2}}
$$

which fails, but not by much. In fact, we shall reduce Theorem 1 to certain perturbations around this estimate (see (5.5) and (5.6), which in turn reduce, on account of the null structure, to some well-known bilinear spacetime estimates of the type first studied by Klainerman and Machedon [22]. For the free wave case, see 3.10] below.

Let us now verify that that the null condition (N1), (N2) is satisfied by (3.7). In fact, since $\Pi_{ \pm}(D)$ does not involve time at all, it suffices to consider spinor fields $\psi(x), \psi^{\prime}(x)$. Replacing $X, Y, Z$ in (3.4) by $\xi, \eta, \zeta \in \mathbb{R}^{3}$, we then have

$$
\left[\left\langle\beta \Pi_{+}(D) \psi, \Pi_{ \pm}(D) \psi^{\prime}\right\rangle\right]^{-}(\xi)=\iint_{\eta+\zeta=\xi}\left\langle\beta \Pi_{+}(\eta) \widehat{\psi}(\eta), \Pi_{ \pm}(-\zeta) \widehat{\psi}^{\prime}(-\zeta)\right\rangle d \eta d \zeta
$$

and since $\Pi_{ \pm}(\xi)^{\dagger}=\Pi_{ \pm}(\xi)$, we obtain

$$
\begin{aligned}
\left\langle\beta \Pi_{+}(\eta) \widehat{\psi}(\eta), \Pi_{ \pm}(-\zeta) \widehat{\psi}^{\prime}(-\zeta)\right\rangle & =\left\langle\Pi_{ \pm}(-\zeta) \beta \Pi_{+}(\eta) \widehat{\psi}(\eta), \widehat{\psi}^{\prime}(-\zeta)\right\rangle \\
& =\left\langle\beta \Pi_{\mp}(-\zeta) \Pi_{+}(\eta) \widehat{\psi}(\eta), \widehat{\psi}^{\prime}(-\zeta)\right\rangle,
\end{aligned}
$$

where in the last step we used the commutation identity (2.3), which inverts the sign.

Thus, we have:

Lemma 1. The symbol of 3.7) is the matrix $\beta \Pi_{\mp}(-\zeta) \Pi_{+}(\eta)$.

The symbol $\beta \Pi_{\mp}(-\zeta) \Pi_{+}(\eta)$ does indeed satisfy the null condition (N1), (N2), by orthogonality of the eigenspaces. In fact, the symbol vanishes to first order in the angle (note that the following lemma can be applied in all cases, i.e., for all combinations of signs, because $\left.\Pi_{+}(-\xi)=\Pi_{-}(\xi)\right)$.

Lemma 2. $\Pi_{+}(\xi) \Pi_{-}(\eta)=O(\theta)$, where $\theta=\angle(\xi, \eta)$. 
Proof.

$$
\begin{aligned}
4 \Pi_{+}(\xi) \Pi_{-}(\eta) & =\left(I+\hat{\xi}_{j} \alpha^{j}\right)\left(I-\hat{\eta}_{k} \alpha^{k}\right) \\
& =I-\hat{\xi}_{j} \hat{\eta}_{k} \alpha^{j} \alpha^{k}+(\hat{\xi}-\hat{\eta}) \cdot \alpha \\
& \left.=(1-\hat{\xi} \cdot \hat{\eta}) I-i \epsilon^{j k l} \hat{\xi}_{j} \hat{\eta}_{k} S^{l}+(\hat{\xi}-\hat{\eta}) \cdot \alpha \quad \text { by (1.4) }\right) \\
& =\underbrace{(1-\hat{\xi} \cdot \hat{\eta})}_{O\left(\theta^{2}\right)} I-i \underbrace{(\hat{\xi} \times \hat{\eta})}_{O(\theta)} \cdot S+\underbrace{(\hat{\xi}-\hat{\eta})}_{O(\theta)} \cdot \alpha
\end{aligned}
$$

where $\hat{\xi} \equiv \xi /|\xi|$ and $S=\left(S^{1}, S^{2}, S^{3}\right)$.

Remark 2. For readers familiar with the standard null forms $Q_{0}, Q_{i j}$ and $Q_{0 j}$, we point out that the factors $1-\hat{\xi} \cdot \hat{\eta}, \hat{\xi} \times \hat{\eta}$ and $\hat{\xi}-\hat{\eta}$ are the symbols of $Q_{0}\left(|D|^{-1} u,|D|^{-1} v\right)$, $Q_{i j}\left(|D|^{-1} u,|D|^{-1} v\right)$ and $Q_{0 j}\left(|D|^{-1} u,|D|^{-1} v\right)$, respectively, in the case of free waves $\square u=\square v=0$.

This concludes the discussion of the null structure in DKG. To illustrate how it is used, let us estimate the left hand side of 3.8) in the important case of free waves $\psi_{ \pm}$ given by (3.5), 3.6). Taking the ++ case for the sake of definiteness, we shall prove

$$
\left\||\square|^{-1 / 2}\left\langle\beta \psi_{+}, \psi_{+}\right\rangle\right\|_{L_{t, x}^{2}} \leq C\left\|\psi_{0}\right\|_{L^{2}}^{2} .
$$

Applying Lemmas 1 and 2, we see that

$$
\begin{aligned}
& \left|\left[|\square|^{-1 / 2}\left\langle\beta \psi_{+}, \psi_{+}\right\rangle\right]^{\sim}(\tau, \xi)\right| \\
& \quad=\frac{1}{\sqrt{|\xi|^{2}-\tau^{2}}}\left|\int_{\mathbb{R}^{3}} \delta(\tau+|\eta|-|\eta-\xi|)\left\langle\beta \Pi_{-}(\eta-\xi) \Pi_{+}(\eta) \widehat{\psi}_{0}(\eta), \widehat{\psi}_{0}(\eta-\xi)\right\rangle d \eta\right| \\
& \quad \leq \frac{C}{\sqrt{|\xi|^{2}-\tau^{2}}} \int_{\mathbb{R}^{3}} \theta \delta(\tau+|\eta|-|\eta-\xi|)\left|\widehat{\psi}_{0}(\eta)\right|\left|\widehat{\psi}_{0}(\eta-\xi)\right| d \eta \\
& \quad \leq C \int_{\mathbb{R}^{3}} \delta(\tau+|\eta|-|\eta-\xi|) \frac{\left|\widehat{\psi}_{0}(\eta)\right|\left|\widehat{\psi}_{0}(\eta-\xi)\right|}{|\eta|^{1 / 2}|\eta-\xi|^{1 / 2}} d \eta,
\end{aligned}
$$

where $\theta=\angle(\eta, \eta-\xi)$ and we used

$$
|\xi|^{2}-\tau^{2}=|\xi|^{2}-(|\eta|-|\eta-\xi|)^{2}=2|\eta||\eta-\xi|(1-\cos \theta) \approx|\eta||\eta-\xi| \theta^{2} .
$$

Here and below, the notation $X \approx Y$ stands for $C^{-1} X \leq Y \leq C X$ where $C>0$ is some absolute constant.

We conclude (going back to physical space and applying Hölder's inequality) that 3.10) reduces to the classical estimate

$$
\left\|e^{ \pm i t|D|} f\right\|_{L^{4}\left(\mathbb{R}^{1+3}\right)} \leq C\|f\|_{\dot{H}^{1 / 2}}
$$

due to Strichartz [35]. 


\section{Some properties of $X^{s, b}$ and $H^{s, b}$}

Here we recall some basic, well-known properties of $X^{s, b}$ and $H^{s, b}$ spaces, needed in the proof of our main result, Theorem 1 . For the convenience of the reader we include short sketches of the proofs in some cases. For more details and further references, see e.g. [27, 36].

We start with $X^{s, b}$, commenting on the more complicated $H^{s, b}$ at the end. The discussion applies to $X^{s, b}$ in general form: Starting from a PDE on $\mathbb{R}^{1+n}$, with any $n \geq 1$, of the form

$$
-i \partial_{t} u=h(D) u
$$

where $h: \mathbb{R}^{n} \rightarrow \mathbb{R}$ and $h(D)$ is the multiplier with symbol $h(\xi)$, one defines $X^{s, b}$ $(s, b \in \mathbb{R})$ via the norm

$$
\|u\|_{X^{s, b}}=\left\|\langle\xi\rangle^{s}\langle\tau-h(\xi)\rangle^{b} \widetilde{u}(\tau, \xi)\right\|_{L_{\tau, \xi}^{2}} .
$$

The cases of interest for us here are $h(\xi)=-|\xi|$, which gives $X_{+}^{s, b}$, and $h(\xi)=|\xi|$, which gives $X_{-}^{s, b}$, but we prefer to keep the general notation (and general dimension) in this section.

Note that $e^{i t h(D)}$ is the free propagator of 4.1. The first important observation is that the elements of $X^{s, b}$ are superpositions of free solutions with $H^{s}$ data, suitably modulated:

Lemma 3. $u \in X^{s, b}$ if and only if there exists $f \in L^{2}\left(\langle\lambda\rangle^{2 b} d \lambda ; H^{s}\left(\mathbb{R}^{n}\right)\right)$ such that

$$
u(t)=\int_{\mathbb{R}} e^{i t \lambda} e^{i t h(D)} f(\lambda) d \lambda \quad\left(H^{s} \text {-valued }\right) .
$$

Moreover, $\|u\|_{X^{s, b}}^{2}=\int_{\mathbb{R}}\|f(\lambda)\|_{H^{s}}^{2}\langle\lambda\rangle^{2 b} d \lambda$.

Proof. The idea is to foliate Fourier space $(\tau, \xi) \in \mathbb{R} \times \mathbb{R}^{n}$ by the surfaces $\tau-h(\xi)=$ const. Define $f(\lambda)$ (a.e.) by $[f(\lambda)]^{-}(\xi)=\widetilde{u}(\lambda+h(\xi), \xi)$. Then

$$
\widetilde{u}(\tau, \xi)=\int \delta(\lambda-\tau+h(\xi)) \widehat{f(\lambda)}(\xi) d \lambda,
$$

which agrees with the spacetime Fourier transform of 4.2 .

From this lemma and the dominated convergence theorem for $H^{s}$-valued integrals, one easily obtains

$$
X^{s, b} \hookrightarrow C_{\mathrm{b}}\left(\mathbb{R} ; H^{s}\right) \quad \text { for } b>1 / 2,
$$

where $\hookrightarrow$ means continuous inclusion. This in turn implies $X^{s, b}\left(S_{T}\right) \hookrightarrow C\left([0, T] ; H^{s}\right)$ for $b>1 / 2$.

Another easy but exceedingly useful consequence of Lemma 3 is that Strichartz type estimates (linear or multilinear) for 4.1p imply corresponding estimates for $X^{s, b}$ : 
Lemma 4. Let $T$ be a multilinear operator $\left(f_{1}(x), \ldots, f_{k}(x)\right) \mapsto T\left(f_{1}, \ldots, f_{k}\right)(x)$ acting in $x$-space. If $T$ satisfies an estimate of the form

$$
\left\|T\left(e^{i t h_{1}(D)} f_{1}, \ldots, e^{i t h_{k}(D)} f_{k}\right)\right\|_{L_{t}^{q} L_{x}^{r}} \leq C\left\|f_{1}\right\|_{H^{s_{1}}} \cdots\left\|f_{k}\right\|_{H^{s_{k}}},
$$

then

$$
\left\|T\left(u_{1}, \ldots, u_{k}\right)\right\|_{L_{t}^{q} L_{x}^{r}} \leq C_{b}\left\|u_{1}\right\|_{X_{1}^{s_{1}, b}} \cdots\left\|u_{k}\right\|_{X_{k}^{s_{k}, b}}
$$

for all $u_{j} \in X_{j}^{s_{j}, b}, 1 \leq j \leq k$, provided $b>1 / 2$. Here $X_{j}^{s, b}$ is defined using the symbol $h_{j}(\xi)$.

Proof. Since $T$ acts only in $x$, not in $t$, the multilinearity gives, using the representation from Lemma 3 .

$$
T\left(u_{1}, \ldots, u_{k}\right)=\int_{\mathbb{R}} \cdots \int_{\mathbb{R}} e^{i t\left(\lambda_{1}+\cdots+\lambda_{k}\right)} T\left(e^{i t h_{1}(D)} f_{1}, \ldots, e^{i t h_{k}(D)} f_{k}\right) d \lambda_{1} \cdots d \lambda_{k} .
$$

Minkowski's integral inequality followed by Cauchy-Schwarz in $d \lambda_{j}$ easily leads to the desired estimate.

For example, the classical Strichartz estimate (3.11) implies $X_{ \pm}^{1 / 2, b} \hookrightarrow L^{4}\left(\mathbb{R}^{1+3}\right)$ for $b>1 / 2$.

Finally, we consider the LWP of the linear Cauchy problem associated to 4.1],

$$
-\left(i \partial_{t}+h(D)\right) u=F(t, x), \quad u(0, x)=f(x),
$$

in the restricted space $X^{s, b}\left(S_{T}\right), b>1 / 2$. In the rest of the paper we impose the condition $T \leq 1$ to avoid having to keep track of the growth of certain constants as $T$ becomes large.

Lemma 5. Let $1 / 2<b \leq 1, s \in \mathbb{R}, 0<T \leq 1$. Also, let $0 \leq \delta \leq 1-b$. Then for all data $F \in X^{s, b-1+\delta}\left(S_{T}\right), f \in H^{s}$, the Cauchy problem 4.4] has a unique solution $u \in X^{s, b}\left(S_{T}\right)$, satisfying the first equation of 4.4 in the sense of $\mathcal{D}^{\prime}\left(S_{T}\right)$. Moreover,

$$
\|u\|_{X^{s, b}\left(S_{T}\right)} \leq C\left(\|f\|_{H^{s}}+T^{\delta}\|F\|_{X^{s, b-1+\delta}\left(S_{T}\right)}\right),
$$

where $C$ only depends on $b$.

Note that in Fourier space, $[\tau-h(\xi)] \widetilde{u}(\tau, \xi)=\widetilde{F}(\tau, \xi)$, so heuristically, [4.5) says that in the time localized case, we can replace the singular symbol $\tau-h(\xi)$ by $\langle\tau-h(\xi)\rangle$, and simply divide out.

In the following proof sketch, we follow closely the argument given in [19], but with a slight modification to get the factor $T^{\delta}$ on the right hand side of 4.5 , which of course is useful in a contraction argument.

Proof of Lemma 5 Start by picking any extension of $F$, which we still denote $F$. Then the problem is to prove 4.5 without the time restriction on the right hand side. Split $u=u_{0}+u_{1}$, where $u_{0}$ is the homogeneous part, $u_{1}$ the inhomogeneous part. Further 


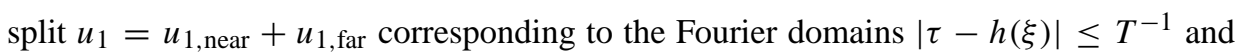
$|\tau-h(\xi)|>T^{-1}$.

First, $\widetilde{u}_{0}(\tau, \xi)=\delta(\tau-h(\xi)) \widehat{f}(\xi)$, hence if $\chi(t)$ is a smooth cut-off function such that $\chi(t)=1$ for $|t| \leq 1$ and $\chi(t)=0$ for $|t| \geq 2$, then $\left\|u_{0}\right\|_{X^{s, b}\left(S_{T}\right)}$ is bounded by

$$
\left\|\chi(t) u_{0}(t, \cdot)\right\|_{X^{s, b}}=\left\|\langle\xi\rangle^{s}\langle\tau-h(\xi)\rangle^{b} \widehat{\chi}(\tau-h(\xi)) \widehat{f}(\xi)\right\|_{L_{\tau, \xi}^{2}}=\|\chi\|_{H^{b}}\|f\|_{H^{s}} .
$$

Next, since (here we use $\mathbb{1}$ to denote the indicator function of the set determined by the condition in the subscript)

$$
\left[u_{1, \mathrm{far}}\right]^{\sim}(\tau, \xi)=\frac{\mathbb{1}_{|\tau-h(\xi)|>T^{-1}}}{\tau-h(\xi)} \widetilde{F}(\tau, \xi),
$$

it follows that $\left\|u_{1, \text { far }}\right\|_{X^{s, b}} \leq C T^{\delta}\|F\|_{X^{s, b-1+\delta}}$.

Finally, Duhamel's principle gives $u_{1 \text {, near }}(t)=\sum_{k=1}^{\infty}\left(t^{k} / k !\right) e^{i t h(D)} g_{k}$, where

$$
\widehat{g}_{k}(\xi)=\int[i(\lambda-h(\xi))]^{k-1} \mathbb{1}_{|\lambda-h(\xi)| \leq T^{-1}} \widetilde{F}(\lambda, \xi) d \lambda .
$$

Using Cauchy-Schwarz in $d \lambda$ one easily obtains $\left\|g_{k}\right\|_{H^{s}} \leq C T^{b-1 / 2+\delta-k}\|F\|_{X^{s, b-1+\delta}}$, so finally,

$$
\begin{aligned}
\left\|u_{1, \text { near }}\right\|_{X^{s, b}\left(S_{T}\right)} & \leq\left\|\chi\left(\frac{t}{T}\right) u_{1, \text { near }}(t, \cdot)\right\|_{X^{s, b}} \\
& \leq C \sum_{k=1}^{\infty} \frac{T^{k}}{k !}\left\|\left(\frac{t}{T}\right)^{k} \chi\left(\frac{t}{T}\right)\right\|_{H_{t}^{b}}\left\|g_{k}\right\|_{H^{s}} \\
& \leq C \sum_{k=1}^{\infty} \frac{T^{k}}{k !}\left(T^{1 / 2-b}\left\|t^{k} \chi(t)\right\|_{H_{t}^{b}}\right)\left(T^{b-1 / 2+\delta-k}\|F\|_{X^{s, b-1+\delta}}\right) \\
& \leq C T^{\delta}\left(\sum_{k=1}^{\infty} \frac{k 2^{k-1}}{k !}\right)\|F\|_{X^{s, b-1+\delta}},
\end{aligned}
$$

since $\left\|t^{k} \chi(t)\right\|_{H_{t}^{b}} \leq\left\|t^{k} \chi(t)\right\|_{H_{t}^{1}} \leq C_{\chi}\left(2^{k}+k 2^{k-1}\right)$ by the support assumption.

To end this section, let us state the analogues of Lemmas 3 for $H^{s, b}$. For $u \in H^{s, b}$ we split $u=u_{+}+u_{-}$corresponding to the Fourier domains $-\tau>0$ and $-\tau<0$, i.e., we set $\widetilde{u}_{ \pm}(\tau, \xi)=\mathbb{1}_{ \pm(-\tau)>0} \widetilde{u}(\tau, \xi)$. (Letting $-\tau$ determine the sign is consistent with the choice of signs in the projections 2.2 , since $-\tau$ corresponds to the energy.) Then $u \in H^{s, b}$ is equivalent to saying that $u_{ \pm} \in X_{ \pm}^{s, b}$, so Lemma 3 applies to give a characterization of $H^{s, b}$ in terms of superimposed free waves. Moreover, $\|u\|_{H^{s, b}}^{2}=\left\|u_{+}\right\|_{X_{+}^{s, b}}^{2}+\left\|u_{-}\right\|_{X_{-}^{s, b}}^{2}$, so Lemma 4 also applies: If $T$ is as in Lemma 4 and the estimate

$$
\left\|T\left(e^{ \pm i t|D|} f_{1}, \ldots, e^{ \pm i t|D|} f_{k}\right)\right\|_{L_{t}^{q} L_{x}^{r}} \leq C\left\|f_{1}\right\|_{H^{s_{1}}} \cdots\left\|f_{k}\right\|_{H^{s_{k}}}
$$

holds, then

$$
\left\|T\left(u_{1}, \ldots, u_{k}\right)\right\|_{L_{t}^{q} L_{x}^{r}} \leq C_{b}\left\|u_{1}\right\|_{H^{s_{1}, b}} \cdots\left\|u_{k}\right\|_{H^{s_{k}, b}}
$$

for all $u_{j} \in H^{s_{j}, b}, 1 \leq j \leq k$, provided $b>1 / 2$. 
Next, consider the Cauchy problem

$$
\square u=F(t, x), \quad u(0, x)=f(x), \quad \partial_{t} u(0, x)=g(x) .
$$

One may think that by rewriting (4.6) as a first order system, Lemma 5 could be applied directly, but if one works with data in inhomogeneous Sobolev spaces, there is a problem with low frequencies. Thus, the following lemma requires a separate proof, which can be found in [33, 34]; see [25] for an earlier version of this estimate (in a slightly different norm, and with $T=1$ ).

Lemma 6. Let $1 / 2<b \leq 1, s \in \mathbb{R}, 0<T \leq 1$ and $0 \leq \delta \leq 1-b$. Then for all data $F \in H^{s-1, b-1+\delta}\left(S_{T}\right), f \in H^{s}$ and $g \in H^{s-1}$, there exists a unique $u \in \mathcal{H}^{s, b}\left(S_{T}\right)$ solving 4.6 on $S_{T}$. Moreover,

$$
\|u\|_{\mathcal{H}^{s, b}\left(S_{T}\right)} \leq C\left(\|f\|_{H^{s}}+\|g\|_{H^{s-1}}+\sigma(T)\|F\|_{H^{s-1, b-1+\delta}\left(S_{T}\right)}\right),
$$

where $\sigma>0$ depends continuously on $T>0$ and satisfies $\lim _{T \rightarrow 0^{+}} \sigma(T)=0$ if $\delta>0$.

\section{Proof of Theorem 1}

We use the iteration scheme ${ }^{2}$ for $k \geq-1$,

$$
\left\{\begin{array}{l}
\left(-i \partial_{t}+|D|\right) \psi_{+}^{k+1}=-M \beta \psi_{-}^{k}+\Pi_{+}(D)\left(\phi^{k} \beta \psi^{k}\right), \\
\left(-i \partial_{t}-|D|\right) \psi_{-}^{k+1}=-M \beta \psi_{+}^{k}+\Pi_{-}(D)\left(\phi^{k} \beta \psi^{k}\right), \\
\square \phi^{k+1}=m^{2} \phi^{k}-\left\langle\beta \psi^{k}, \psi^{k}\right\rangle,
\end{array}\right.
$$

where $\psi^{k}=\psi_{+}^{k}+\psi_{-}^{k}$ and $\psi_{ \pm}^{-1}, \phi^{-1} \equiv 0$. The initial data are

$$
\psi_{ \pm}^{k}(0, x)=\Pi_{ \pm}(D) \psi_{0}(x), \quad \phi^{k}(0, x)=\phi_{0}(x), \quad \partial_{t} \phi^{k}(0, x)=\phi_{1}(x)
$$

where $\psi_{0} \in H^{\varepsilon},\left(\phi_{0}, \phi_{1}\right) \in H^{1 / 2+\varepsilon} \times H^{-1 / 2+\varepsilon}$ for some $0<\varepsilon \leq 1 / 2$. Consider $\varepsilon$ fixed, and let $\varepsilon^{\prime}>0$ denote a sufficiently small number, depending on $\varepsilon$. We iterate in the spaces

$$
\psi_{ \pm}^{k} \in X_{ \pm}^{\varepsilon, 1 / 2+\varepsilon^{\prime}}\left(S_{T}\right), \quad \phi^{k} \in \mathcal{H}^{1 / 2+\varepsilon, 1 / 2+\varepsilon^{\prime}}\left(S_{T}\right)
$$

where $S_{T}=(0, T) \times \mathbb{R}^{3}$ for some $0<T \leq 1$ to be chosen sufficiently small depending on the size of

$$
\mathcal{I}_{0}=\left\|\psi_{0}\right\|_{H^{\varepsilon}}+\left\|\phi_{0}\right\|_{H^{1 / 2+\varepsilon}}+\left\|\phi_{1}\right\|_{H^{-1 / 2+\varepsilon}}
$$

\footnotetext{
2 It may be more natural to include the mass terms in the operators on the left hand sides, but this would require generalizing the Klainerman-Machedon type estimates to the massive operators; while this can certainly be done (and in some cases has been done), it is not something we wish to undertake in the present paper. In any event, putting the linear mass terms on the right hand sides is harmless as far as the local in time contraction is concerned.
} 
In the estimates that follow, $\lesssim$ stands for $\leq$ up to a multiplicative constant $C$ which may depend on $\varepsilon$ but is independent of $T$, and $\sigma(T)$ denotes a positive, continuous function of $0<T \leq 1$ such that $\lim _{T \rightarrow 0^{+}} \sigma(T)=0$.

By induction, $\Pi_{ \pm}(D) \psi_{ \pm}^{k}=\psi_{ \pm}^{k}$ for all $k$, hence

$$
\psi^{k}=\Pi_{+}(D) \psi_{+}^{k}+\Pi_{-}(D) \psi_{-}^{k} .
$$

By Lemmas 5 and 6 .

$$
\begin{aligned}
& \left\|\psi_{ \pm}^{k+1}\right\|_{X_{ \pm}^{\varepsilon, 1 / 2+\varepsilon^{\prime}}\left(S_{T}\right)} \lesssim \mathcal{I}_{0}+M\left\|\psi_{\mp}^{k}\right\|_{L^{2}\left([0, T] ; H^{\varepsilon}\right)} \\
& +\sigma(T)\left\|\Pi_{ \pm}(D)\left(\phi^{k} \beta \psi^{k}\right)\right\|_{X_{ \pm}^{\varepsilon,-1 / 2+2 \varepsilon^{\prime}}}{\left(S_{T}\right)}, \\
& \left\|\phi^{k+1}\right\|_{\mathcal{H}^{1 / 2+\varepsilon, 1 / 2+\varepsilon^{\prime}}\left(S_{T}\right)} \lesssim \mathcal{I}_{0}+m^{2}\left\|\phi^{k}\right\|_{L^{2}\left([0, T] ; H^{-1 / 2+\varepsilon}\right)} \\
& +\sigma(T)\left\|\left\langle\beta \psi^{k}, \psi^{k}\right\rangle\right\|_{H^{-1 / 2+\varepsilon,-1 / 2+2 \varepsilon^{\prime}}\left(S_{T}\right)},
\end{aligned}
$$

so in view of (5.1), the key is to establish the general estimates

$$
\begin{aligned}
&\left\|\Pi_{ \pm}(D)\left(\phi \beta \Pi_{[ \pm]}(D) \psi\right)\right\|_{X_{ \pm}^{\varepsilon,-1 / 2+2 \varepsilon^{\prime}}} \lesssim\|\phi\|_{H^{1 / 2+\varepsilon, 1 / 2+\varepsilon^{\prime}}}\|\psi\|_{X_{[ \pm]}^{\varepsilon, 1 / 2+\varepsilon^{\prime}}} \\
&\left\|\left\langle\beta \Pi_{[ \pm]}(D) \psi, \Pi_{ \pm}(D) \psi^{\prime}\right\rangle\right\|_{H^{-1 / 2+\varepsilon,-1 / 2+2 \varepsilon^{\prime}}} \lesssim\|\psi\|_{X_{[ \pm]}^{\varepsilon, 1 / 2+\varepsilon^{\prime}}\left\|\psi^{\prime}\right\|_{X_{ \pm}^{\varepsilon, 1 / 2+\varepsilon^{\prime}}}}
\end{aligned}
$$

for $\varepsilon^{\prime}>0$ sufficiently small depending on $\varepsilon$. Here \pm and $[ \pm]$ denote independent signs. Also, in (5.4) and (5.5) the norms are not restricted to $S_{T}$, but they are applied to extensions of the iterates $\phi^{k}, \psi^{k}$. Taking infima over all extensions, one then obtains, from (5.2)-5.5,

$$
A_{k+1}(T) \lesssim \mathcal{I}_{0}+\sigma(T) P\left(A_{k}(T)\right)
$$

where

$$
A_{k}(T)=\left\|\phi^{k}\right\|_{\mathcal{H}^{1 / 2+\varepsilon, 1 / 2+\varepsilon^{\prime}\left(S_{T}\right)}}+\sum_{ \pm}\left\|\psi_{ \pm}^{k}\right\|_{X_{ \pm}^{\varepsilon, 1 / 2+\varepsilon^{\prime}}\left(S_{T}\right)}
$$

and $P$ is a polynomial such that $P(0)=0$. Here we have also used the fact that

$$
\|\psi\|_{L^{2}\left([0, T] ; H^{\varepsilon}\right)} \leq T^{1 / 2}\|\psi\|_{L^{\infty}\left([0, T] ; H^{\varepsilon}\right)} \lesssim T^{1 / 2}\|\psi\|_{X_{ \pm}^{\varepsilon, 1 / 2+\varepsilon^{\prime}}\left(S_{T}\right)}
$$

and similarly for the term $\left\|\phi^{k}\right\|_{L^{2}\left([0, T] ; H^{-1 / 2+\varepsilon}\right)}$ in 5.3 .

In view of the multilinearity of the nonlinear terms in the system, one obtains similar estimates for the difference of subsequent iterates, and the standard contraction argument then gives local existence and uniqueness (in the iteration space) for $0<T \leq 1$ sufficiently small depending on $\mathcal{I}_{0}$.

Thus, we have reduced to proving (5.4) and (5.5). But by duality, (5.4) is equivalent to

$$
\iint\left\langle\Pi_{ \pm}(D)\left(\phi \beta \Pi_{[ \pm]}(D) \psi\right), \psi^{\prime}\right\rangle d t d x \lesssim\|\phi\|_{H^{1 / 2+\varepsilon, 1 / 2+\varepsilon^{\prime}}}\|\psi\|_{X_{[ \pm]}^{\varepsilon, 1 / 2+\varepsilon^{\prime}}}\left\|\psi^{\prime}\right\|_{X_{ \pm}^{-\varepsilon, 1 / 2-2 \varepsilon^{\prime}}}
$$


and since

$$
\begin{aligned}
\iint\left\langle\Pi_{ \pm}(D)\left(\phi \beta \Pi_{[ \pm]}(D) \psi\right), \psi^{\prime}\right\rangle d t d x \\
\quad=\iint\left\langle\phi \beta \Pi_{[ \pm]}(D) \psi, \Pi_{ \pm}(D) \psi^{\prime}\right\rangle d t d x \\
\quad=\iint \phi\left\langle\beta \Pi_{[ \pm]}(D) \psi, \Pi_{ \pm}(D) \psi^{\prime}\right\rangle d t d x \\
\leq\|\phi\|_{H^{1 / 2+\varepsilon, 1 / 2+\varepsilon^{\prime}}}\left\|\left\langle\beta \Pi_{[ \pm]}(D) \psi, \Pi_{ \pm}(D) \psi^{\prime}\right\rangle\right\|_{H^{-1 / 2-\varepsilon,-1 / 2-\varepsilon^{\prime}}},
\end{aligned}
$$

we conclude that (5.4) reduces to an estimate similar to 5.5):

$$
\left\|\left\langle\beta \Pi_{[ \pm]}(D) \psi, \Pi_{ \pm}(D) \psi^{\prime}\right\rangle\right\|_{H^{-1 / 2-\varepsilon,-1 / 2-2 \varepsilon^{\prime}}} \lesssim\|\psi\|_{X_{[ \pm]}^{\varepsilon, 1 / 2+\varepsilon^{\prime}}}\left\|\psi^{\prime}\right\|_{X_{ \pm}^{-\varepsilon, 1 / 2-2 \varepsilon^{\prime}}}
$$

Note that both (5.5) and (5.6) are perturbations around the false estimate (3.8). In fact, using the null structure we shall reduce (5.5) and 5.6 to some well-known bilinear spacetime estimates of Klainerman-Machedon type for products of free waves. Specifically, we need the following generalization of the classical $L^{4}$ estimate 3.11 of Strichartz.

Theorem 2 ([22, 26, 16]). Let $s_{1}, s_{2}, s_{3} \in \mathbb{R}$. The estimate

$$
\left\||D|^{-s_{3}}(u v)\right\|_{L^{2}\left(\mathbb{R}^{1+3}\right)} \leq C_{s_{1}, s_{2}, s_{3}}\left\|u_{0}\right\|_{\dot{H}^{s_{1}}}\left\|v_{0}\right\|_{\dot{H}^{s_{2}}}
$$

holds for free waves $u(t)=e^{ \pm i t|D|} u_{0}, v(t)=e^{ \pm i t|D|} v_{0}$ if and only if

$$
s_{1}+s_{2}+s_{3}=1, \quad s_{1}, s_{2}, s_{3}<1, \quad s_{1}+s_{2}>1 / 2 .
$$

From this and Lemma 4 we obtain

$$
H^{s_{1}, b} \cdot H^{s_{2}, b} \rightarrow H^{-s_{3}, 0} \quad \text { for } b>1 / 2 \text { and } s_{1}, s_{2}, s_{3} \geq 0 \text { satisfying (5.7). }
$$

Here we use the following notation: If $X, Y, Z$ are normed spaces of functions, the statement $X \cdot Y \rightarrow Z$ means that the bilinear estimate $\|u v\|_{Z} \leq C\|u\|_{X}\|v\|_{Y}$ holds for some constant $C$.

By interpolation between (5.8) and the estimate

$$
L^{2} \cdot H^{0, b} \rightarrow H^{-N, 0} \quad \text { for } b>1 / 2, N>3 / 2,
$$

which by duality is equivalent to $H^{N, 0} \cdot H^{0, b} \rightarrow L^{2}$ and therefore follows from Hölder's inequality $L_{t}^{\infty} L_{x}^{2} \cdot L_{t}^{2} L_{x}^{\infty} \rightarrow L^{2}$ and Sobolev embedding, it is easy to prove (see the next section) the estimates

$$
\begin{aligned}
H^{0,1 / 2-\delta} \cdot H^{1 / 2+\varepsilon, b} & \rightarrow H^{-1 / 2,0}, \\
H^{0,1 / 2-\delta} \cdot H^{1 / 2, b} & \rightarrow H^{-1 / 2-\varepsilon, 0}, \\
H^{1 / 2-\varepsilon, 1 / 2-\delta} \cdot H^{1 / 2+\varepsilon, b} & \rightarrow H^{-\varepsilon, 0}, \\
H^{1 / 2-\varepsilon, 1 / 2-\delta} \cdot H^{\varepsilon, b} & \rightarrow H^{-1 / 2-\varepsilon, 0}, \\
H^{-\varepsilon, 1 / 2-\delta} \cdot H^{1 / 2+\varepsilon, b} & \rightarrow H^{-1 / 2-\varepsilon, 0},
\end{aligned}
$$

for all $b>1 / 2, \varepsilon>0$ and sufficiently small $\delta>0$, depending on $\varepsilon$. 
We now turn to the proofs of (5.5) and (5.6). It suffices to consider the case where the $\operatorname{sign}[ \pm]$ is a + . Throughout the rest of this section, we set $b=1 / 2+\varepsilon^{\prime}$; recall that $\varepsilon^{\prime}>0$ denotes a sufficiently small number, depending on $\varepsilon$.

\subsection{Proof of 5.5}

Using Lemmas 1 and 2, we see that 5.5 reduces to proving

$$
\begin{aligned}
&\left\|\frac{1}{\langle\xi\rangle^{1 / 2-\varepsilon}\langle|\tau|-|\xi|\rangle^{1 / 2-2 \varepsilon^{\prime}}} \iint \theta_{ \pm}|\tilde{\psi}(\lambda, \eta)|\left|\tilde{\psi}^{\prime}(\lambda-\tau, \eta-\xi)\right| d \lambda d \eta\right\|_{L_{\tau, \xi}^{2}} \\
& \lesssim\|\psi\|_{X_{+}^{\varepsilon, 1 / 2+\varepsilon^{\prime}}\left\|\psi^{\prime}\right\|_{X_{ \pm}^{\varepsilon, 1 / 2+\varepsilon^{\prime}}}}
\end{aligned}
$$

where

$$
\theta_{ \pm} \equiv \angle(\eta, \pm(\eta-\xi))
$$

We claim that

$$
\theta_{+}^{2} \approx \frac{|\xi| r_{+}}{|\eta||\eta-\xi|}, \quad \theta_{-}^{2} \approx \frac{(|\eta|+|\eta-\xi|) r_{-}}{|\eta||\eta-\xi|}
$$

where

$$
r_{+} \equiv|\xi|-|| \eta|-| \eta-\xi||, \quad r_{-} \equiv|\eta|+|\eta-\xi|-|\xi| .
$$

To prove the estimate for $\theta_{+}^{2}$, one writes

$$
\frac{|\xi| r_{+}}{|\eta||\eta-\xi|} \approx \frac{(|\xi|+|| \eta|-| \eta-\xi||) r_{+}}{|\eta||\eta-\xi|}=\frac{|\xi|^{2}-|| \eta|-| \eta-\xi||^{2}}{|\eta||\eta-\xi|}=2\left(1-\cos \theta_{+}\right),
$$

and the estimate for $\theta_{-}^{2}$ is proved in a similar way.

Assuming as we may that $\widetilde{\psi}, \widetilde{\psi}^{\prime} \geq 0$, and suppressing the arguments of these functions to keep the notation manageable, we thus reduce 5.15 to

$$
\begin{aligned}
\left\|\frac{|\xi|^{\varepsilon}}{\langle|\tau|-|\xi|\rangle^{1 / 2-2 \varepsilon^{\prime}}} \iint \frac{r_{+}^{1 / 2}}{|\eta|^{1 / 2}|\eta-\xi|^{1 / 2}} \tilde{\psi} \tilde{\psi}^{\prime} d \lambda d \eta\right\|_{L_{\tau, \xi}^{2}} & \lesssim\|\psi\|_{X_{+}^{\varepsilon, 1 / 2+\varepsilon^{\prime}}}\left\|\psi^{\prime}\right\|_{X_{+}^{\varepsilon, 1 / 2+\varepsilon^{\prime}}}
\end{aligned}
$$

and

$$
\begin{aligned}
\left\|\frac{1}{\langle\xi\rangle^{1 / 2-\varepsilon}\langle|\tau|-|\xi|\rangle^{1 / 2-2 \varepsilon^{\prime}}} \iint \frac{r_{-}^{1 / 2}}{\min (|\eta|,|\eta-\xi|)^{1 / 2}} \widetilde{\psi} \widetilde{\psi}^{\prime} d \lambda d \eta\right\|_{L_{\tau, \xi}^{2}} \\
\lesssim\|\psi\|_{X_{+}^{\varepsilon, 1 / 2+\varepsilon^{\prime}}}\left\|\psi^{\prime}\right\|_{X_{-}^{\varepsilon, 1 / 2+\varepsilon^{\prime}}}
\end{aligned}
$$

Now we apply the following:

Lemma 7. $r_{ \pm} \lesssim|| \tau|-| \xi||+|\lambda+| \eta||+|\lambda-\tau \pm| \eta-\xi||$. 
Proof. If $\tau \geq 0$, we estimate

$$
r_{+} \leq|\xi|+|\eta|-|\eta-\xi|=|\xi|-|\tau|+\lambda+|\eta|+\tau-\lambda-|\eta-\xi|,
$$

while if $\tau<0$ we use

$$
r_{+} \leq|\xi|-|\eta|+|\eta-\xi|=|\xi|-|\tau|-\lambda-|\eta|-\tau+\lambda+|\eta-\xi| .
$$

To handle $r_{-}$we write

$$
r_{-}=\lambda+|\eta|+\tau-\lambda+|\eta-\xi|-\tau-|\xi| .
$$

If $\tau<0$, this equals $\lambda+|\eta|+\tau-\lambda+|\eta-\xi|+|\tau|-|\xi|$, while if $\tau \geq 0$ it is $\leq$ $\lambda+|\eta|+\tau-\lambda+|\eta-\xi|$.

We also need

$$
r_{ \pm} \leq 2 \min (|\eta|,|\eta-\xi|)
$$

which follows from the triangle inequality. Combining this with Lemma 7 we get

$$
r_{ \pm}^{1 / 2} \lesssim|| \tau|-| \xi||^{1 / 2-2 \varepsilon^{\prime}} \min (|\eta|,|\eta-\xi|)^{2 \varepsilon^{\prime}}+|\lambda+| \eta||^{1 / 2}+|\lambda-\tau \pm| \eta-\xi||^{1 / 2}
$$

Moreover, by symmetry we may assume $|\eta| \geq|\eta-\xi|$ in 5.16) and 5.17. Hence 5.16 reduces to proving

$$
I_{j}^{+} \lesssim\|\psi\|_{X_{+}^{\varepsilon, 1 / 2+\varepsilon^{\prime}}}\left\|\psi^{\prime}\right\|_{X_{+}^{\varepsilon, 1 / 2+\varepsilon^{\prime}}}
$$

for $j=1,2,3$, where

$$
\begin{aligned}
& I_{1}^{+}=\left\|\iint \frac{\tilde{\psi} \widetilde{\psi}^{\prime}}{|\eta|^{1 / 2-\varepsilon}|\eta-\xi|^{1 / 2-2 \varepsilon^{\prime}}} d \lambda d \eta\right\|_{L_{\tau, \xi}^{2}}, \\
& I_{2}^{+}=\left\|\frac{1}{\langle|\tau|-|\xi|\rangle^{1 / 2-2 \varepsilon^{\prime}}} \iint \frac{|\lambda+| \eta||^{1 / 2} \widetilde{\psi} \widetilde{\psi}^{\prime}}{|\eta|^{1 / 2-\varepsilon}|\eta-\xi|^{1 / 2}} d \lambda d \eta\right\|_{L_{\tau, \xi}^{2}}, \\
& I_{3}^{+}=\left\|\frac{1}{\langle|\tau|-|\xi|\rangle^{1 / 2-2 \varepsilon^{\prime}}} \iint \frac{\tilde{\psi}|\lambda-\tau+| \eta-\left.\xi\right|^{1 / 2} \widetilde{\psi}^{\prime}}{|\eta|^{1 / 2-\varepsilon}|\eta-\xi|^{1 / 2}} d \lambda d \eta\right\|_{L_{\tau, \xi}^{2}},
\end{aligned}
$$

and 5.17 reduces to proving

$$
I_{j}^{-} \lesssim\|\psi\|_{X_{+}^{\varepsilon, 1 / 2+\varepsilon^{\prime}}}\left\|\psi^{\prime}\right\|_{X_{-}^{\varepsilon, 1 / 2+\varepsilon^{\prime}}}
$$

for $j=1,2,3$, where

$$
\begin{aligned}
& I_{1}^{-}=\left\|\frac{1}{\langle\xi\rangle^{1 / 2-\varepsilon}} \iint \frac{\tilde{\psi} \tilde{\psi}^{\prime}}{|\eta-\xi|^{1 / 2-2 \varepsilon^{\prime}}} d \lambda d \eta\right\|_{L_{\tau, \xi}^{2}}, \\
& I_{2}^{-}=\left\|\frac{1}{\langle\xi\rangle^{1 / 2-\varepsilon}\langle|\tau|-|\xi|\rangle^{1 / 2-2 \varepsilon^{\prime}}} \iint \frac{|\lambda+| \eta||^{1 / 2} \widetilde{\psi} \widetilde{\psi}^{\prime}}{|\eta-\xi|^{1 / 2}} d \lambda d \eta\right\|_{L_{\tau, \xi}^{2}}, \\
& I_{3}^{-}=\left\|\frac{1}{\langle\xi\rangle^{1 / 2-\varepsilon}\langle|\tau|-|\xi|\rangle^{1 / 2-2 \varepsilon^{\prime}}} \iint \frac{\tilde{\psi}|\lambda-\tau-| \eta-\xi||^{1 / 2} \widetilde{\psi}^{\prime}}{|\eta-\xi|^{1 / 2}} d \lambda d \eta\right\|_{L_{\tau, \xi}^{2}} .
\end{aligned}
$$


The estimates for $I_{j}^{+}, j=1,2,3$, reduce to, respectively,

$$
\begin{aligned}
X_{+}^{1 / 2, b} \cdot X_{+}^{1 / 2+\varepsilon-2 \varepsilon^{\prime}, b} & \rightarrow L^{2} \\
X_{+}^{1 / 2,0} \cdot X_{+}^{1 / 2+\varepsilon, b} & \rightarrow H^{0,-1 / 2+2 \varepsilon^{\prime}} \\
X_{+}^{1 / 2, b} \cdot X_{+}^{1 / 2+\varepsilon, 0} & \rightarrow H^{0,-1 / 2+2 \varepsilon^{\prime}},
\end{aligned}
$$

and the estimates for $I_{j}^{-}, j=1,2,3$, reduce to

$$
\begin{aligned}
X_{+}^{\varepsilon, b} \cdot X_{-}^{1 / 2+\varepsilon-2 \varepsilon^{\prime}, b} & \rightarrow H^{-1 / 2+\varepsilon, 0}, \\
X_{+}^{\varepsilon, 0} \cdot X_{-}^{1 / 2+\varepsilon, b} & \rightarrow H^{-1 / 2+\varepsilon,-1 / 2+2 \varepsilon^{\prime}}, \\
X_{+}^{\varepsilon, b} \cdot X_{-}^{1 / 2+\varepsilon, 0} & \rightarrow H^{-1 / 2+\varepsilon,-1 / 2+2 \varepsilon^{\prime}} .
\end{aligned}
$$

Recall that we assume $b=1 / 2+\varepsilon^{\prime}$ throughout this section.

Since in general $\|u\|_{H^{s, b}} \leq\|u\|_{X_{ \pm}^{s, b}}$ for $b \geq 0$, we may in fact replace all the $X_{ \pm}^{s, b}$ on the left hand sides by $H^{s, b}$. (The information encoded in the signs has already been made use of through the null structure.) Using also duality, we thus reduce to

$$
\begin{aligned}
H^{1 / 2, b} \cdot H^{1 / 2+\varepsilon-2 \varepsilon^{\prime}, b} & \rightarrow L^{2}, \\
H^{0,1 / 2-2 \varepsilon^{\prime}} \cdot H^{1 / 2+\varepsilon, b} & \rightarrow H^{-1 / 2,0}, \\
H^{1 / 2, b} \cdot H^{0,1 / 2-2 \varepsilon^{\prime}} & \rightarrow H^{-1 / 2-\varepsilon, 0},
\end{aligned}
$$

and

$$
\begin{aligned}
H^{\varepsilon, b} \cdot H^{1 / 2+\varepsilon-2 \varepsilon^{\prime}, b} & \rightarrow H^{-1 / 2+\varepsilon, 0}, \\
H^{1 / 2-\varepsilon, 1 / 2-2 \varepsilon^{\prime}} \cdot H^{1 / 2+\varepsilon, b} & \rightarrow H^{-\varepsilon, 0}, \\
H^{\varepsilon, b} \cdot H^{1 / 2-\varepsilon, 1 / 2-2 \varepsilon^{\prime}} & \rightarrow H^{-1 / 2-\varepsilon, 0} .
\end{aligned}
$$

All these estimates are true for $\varepsilon^{\prime}>0$ sufficiently small, by 5.8 and 5.10 -5.14 .

\subsection{Proof of (5.6)}

Proceeding as in the proof of 5.5 , we reduce to

$$
\begin{aligned}
&\left\|\frac{|\xi|^{\varepsilon}}{\langle|\tau|-|\xi|\rangle^{1 / 2+\varepsilon^{\prime}}} \iint \frac{r_{+}^{1 / 2}}{|\eta|^{1 / 2}|\eta-\xi|^{1 / 2}} \widetilde{\psi} \widetilde{\psi}^{\prime} d \lambda d \eta\right\|_{L_{\tau, \xi}^{2}} \\
& \lesssim\|\psi\|_{X_{+}^{\varepsilon, 1 / 2+\varepsilon^{\prime}}}\left\|\psi^{\prime}\right\|_{X_{+}^{-\varepsilon, 1 / 2-2 \varepsilon^{\prime}}}
\end{aligned}
$$


and

$$
\begin{aligned}
\left\|\frac{1}{\langle\xi\rangle^{1 / 2+\varepsilon}\langle|\tau|-|\xi|\rangle^{1 / 2+\varepsilon^{\prime}}} \iint \frac{r_{-}^{1 / 2}}{\min (|\eta|,|\eta-\xi|)^{1 / 2}} \widetilde{\psi} \widetilde{\psi}^{\prime} d \lambda d \eta\right\|_{L_{\tau, \xi}^{2}} \\
\lesssim\|\psi\|_{X_{+}^{\varepsilon, 1 / 2+\varepsilon^{\prime}}}\left\|\psi^{\prime}\right\|_{X_{-}^{-\varepsilon, 1 / 2-2 \varepsilon^{\prime}}}
\end{aligned}
$$

By Lemma 7 and 5.18 ,

$$
r_{ \pm}^{1 / 2} \lesssim|| \tau|-| \xi||^{1 / 2}+|\lambda+| \eta||^{1 / 2}+|\lambda-\tau \pm| \eta-\xi||^{1 / 2-2 \varepsilon^{\prime}} \min (|\eta|,|\eta-\xi|)^{2 \varepsilon^{\prime}},
$$

hence 5.25 reduces to (recall that $b=1 / 2+\varepsilon^{\prime}$ )

$$
\begin{aligned}
H^{1 / 2+\varepsilon, b} \cdot H^{1 / 2-\varepsilon, 1 / 2-\varepsilon^{\prime}} & \rightarrow H^{-\varepsilon, 0}, \\
H^{1 / 2+\varepsilon, 0} \cdot H^{1 / 2-\varepsilon, 1 / 2-\varepsilon^{\prime}} & \rightarrow H^{-\varepsilon,-b}, \\
H^{1 / 2+\varepsilon-2 \varepsilon^{\prime}, b} \cdot H^{1 / 2-\varepsilon-2 \varepsilon^{\prime}, 0} & \rightarrow H^{-\varepsilon,-b},
\end{aligned}
$$

which follow from, respectively, 5.12, 5.13) (via duality) and (5.8) (also via duality). Now consider (5.26). Assuming first $|\eta| \leq|\eta-\xi|$ we reduce to

$$
\begin{gathered}
H^{1 / 2+\varepsilon, b} \cdot H^{-\varepsilon, 1 / 2-2 \varepsilon^{\prime}} \rightarrow H^{-1 / 2-\varepsilon, 0}, \\
H^{1 / 2+\varepsilon, 0} \cdot H^{-\varepsilon, 1 / 2-2 \varepsilon^{\prime}} \rightarrow H^{-1 / 2-\varepsilon,-b}, \\
H^{1 / 2+\varepsilon-2 \varepsilon^{\prime}, b} \cdot H^{-\varepsilon, 0} \rightarrow H^{-1 / 2-\varepsilon,-b},
\end{gathered}
$$

while in the case $|\eta| \geq|\eta-\xi|$ we get

$$
\begin{aligned}
& H^{\varepsilon, b} \cdot H^{1 / 2-\varepsilon, 1 / 2-2 \varepsilon^{\prime}} \rightarrow H^{-1 / 2-\varepsilon, 0}, \\
& H^{\varepsilon, 0} \cdot H^{1 / 2+-\varepsilon, 1 / 2-2 \varepsilon^{\prime}} \rightarrow H^{-1 / 2-\varepsilon,-b}, \\
& H^{\varepsilon, b} \cdot H^{1 / 2-\varepsilon-2 \varepsilon^{\prime}, 0} \rightarrow H^{-1 / 2-\varepsilon,-b} .
\end{aligned}
$$

All these reduce (possibly via duality) to 5.8 or $(5.10)-(5.14)$.

\section{Proof of (5.10)-(5.14)}

All these follow by interpolation between (5.9) and various special cases of (5.8). Fix $\varepsilon>0, b>1 / 2, N>3 / 2$. The number $\delta>0$ will be chosen sufficiently small, depending on $\varepsilon$.

For 5.10 we interpolate between

$$
\begin{aligned}
H^{0,1 / 2+\delta} \cdot H^{1 / 2+\varepsilon, b} & \rightarrow H^{-1 / 2+\varepsilon, 0}, \\
L^{2} \cdot H^{1 / 2+\varepsilon, b} & \rightarrow H^{-N, 0} .
\end{aligned}
$$

This gives

$$
H^{0,(1-\theta)(1 / 2+\delta)} \cdot H^{1 / 2+\varepsilon, b} \rightarrow H^{(1-\theta)(-1 / 2+\varepsilon)-\theta N, 0}
$$


for $0 \leq \theta \leq 1$. First choose $\theta>0$ so small that $(1-\theta)(-1 / 2+\varepsilon)-\theta N \geq-1 / 2$. Then choose $\delta>0$ so small that $(1-\theta)(1 / 2+\delta) \leq 1 / 2-\delta$.

For (5.11) we interpolate between

$$
\begin{aligned}
H^{0,1 / 2-\delta} \cdot H^{1 / 2+\varepsilon^{\prime}, b} & \rightarrow H^{-1 / 2,0}, \\
H^{0,1 / 2-\delta} \cdot H^{0, b} & \rightarrow H^{-N, 0},
\end{aligned}
$$

the first of which is just [5.10), for $\varepsilon^{\prime}, \delta>0$ to be chosen sufficiently small, depending on $\varepsilon$. This gives

$$
H^{0,1 / 2-\delta} \cdot H^{(1-\theta)\left(1 / 2+\varepsilon^{\prime}\right), b} \rightarrow H^{(1-\theta)(-1 / 2)-\theta N, 0} .
$$

First choose $\theta>0$ so small that $(1-\theta)(-1 / 2)-\theta N \geq-1 / 2-\varepsilon$. Then choose $\varepsilon^{\prime}>0$ so small that $(1-\theta)\left(1 / 2+\varepsilon^{\prime}\right) \leq 1 / 2$.

For (5.12) interpolate between

$$
\begin{aligned}
H^{1 / 2-\varepsilon, 1 / 2+\delta} \cdot H^{1 / 2+\varepsilon, b} & \rightarrow L^{2}, \\
H^{1 / 2-\varepsilon, 0} \cdot H^{1 / 2+\varepsilon, b} & \rightarrow H^{-N, 0} .
\end{aligned}
$$

Thus,

$$
H^{1 / 2-\varepsilon,(1-\theta)(1 / 2+\delta)} \cdot H^{1 / 2+\varepsilon, b} \rightarrow H^{-\theta N, 0}
$$

for $0 \leq \theta \leq 1$. First choose $\theta>0$ so small that $\theta N \leq \varepsilon$. Then choose $\delta>0$ so small that $(1-\theta)(1 / 2+\delta) \leq 1 / 2-\delta$.

To prove 5.13) we interpolate between

$$
\begin{aligned}
H^{1 / 2-\varepsilon, 1 / 2+\delta} \cdot H^{\varepsilon, b} & \rightarrow H^{-1 / 2,0}, \\
H^{1 / 2-\varepsilon, 0} \cdot H^{\varepsilon, b} & \rightarrow H^{-N, 0},
\end{aligned}
$$

yielding

$$
H^{1 / 2-\varepsilon,(1-\theta)(1 / 2+\delta)} \cdot H^{\varepsilon, b} \rightarrow H^{(1-\theta)(-1 / 2)-\theta N, 0} .
$$

First choose $\theta>0$ so small that $(1-\theta)(1 / 2)+\theta N \leq 1 / 2+\varepsilon$. Then choose $\delta>0$ so small that $(1-\theta)(1 / 2+\delta) \leq 1 / 2-\delta$.

Finally, (5.14) reduces to (5.11) or (5.12), depending on whether the frequency interactions are of type high-high or high-low/low-high in the product on the left hand side.

Acknowledgments. The last author was supported by the Research Council of Norway, project no. 160192/V30, "PDE and Harmonic Analysis". 


\section{References}

[1] Bachelot, A.: Problème de Cauchy pour des systèmes hyperboliques semi-linéaires. Ann. Inst. H. Poincaré Anal. Non Linéaire 1, 453-478 (1984) Zbl 0566.35068 MR 0778979

[2] Bachelot, A.: Problème de Cauchy globale pour des systèmes de Dirac-Klein-Gordon. Ann. Inst. H. Poincaré Phys. Théor. 48, 387-422 (1988) Zbl 0672.35071 MR 0969173

[3] Bachelot, A.: Global existence of large amplitude solutions for Dirac-Klein-Gordon systems in Minkowski space. In: Nonlinear Hyperbolic Problems (Bordeaux, 1988), Lecture Notes in Math. 1402, Springer, Berlin, 99-113 (1989) Zbl 0787.35097, 0705.35118 MR 1033278. 1215780

[4] Beals, R., Bezard, M.: Low regularity local solutions for field equations. Comm. Partial Differential Equations 21, 79-124 (1996) Zbl 0852.35098 MR 1373766

[5] Bourgain, J.: Fourier transform restriction phenomena for certain lattice subsets and applications to nonlinear evolution equations I, II. Geom. Funct. Anal. 3, 107-156 and 209-262 (1993) Zbl 0787.35097, 0787.35098 MR 1209299. 1215780

[6] Bournaveas, N.: Local existence of energy class solutions for the Dirac-Klein-Gordon equations Comm. Partial Differential Equations 24, 1167-1193 (1999) Zbl 0931.35134 MR 1697486

[7] Bournaveas, N.: A new proof of global existence for the Dirac-Klein-Gordon equations in one space dimension. J. Funct. Anal. 173, 203-213 (2000) Zbl 0953.35003 MR 1760283

[8] Bournaveas, N.: Low regularity solutions of the Dirac-Klein-Gordon equations in two space dimensions. Comm. Partial Differential Equations 26, 1345-1366 (2001) Zbl 0991.35093 MR 1855281

[9] Chadam, J. M.: Global solutions of the Cauchy problem for the (classical) coupled MaxwellDirac equations in one space dimension. J. Funct. Anal. 13, 173-184 (1973) Zbl 0264.35058 MR 0368640

[10] Chadam, J. M., Glassey, R.: On certain global solutions of the Cauchy problem for the (classical) coupled Klein-Gordon-Dirac equations in one and three space dimensions. Arch. Ration. Mech. Anal. 54, 223-237 (1974) Zbl 0285.35042 MR 0369952

[11] Choquet-Bruhat, Y.: Solution globale des équations de Maxwell-Dirac-Klein-Gordon., Rend. Circ. Mat. Palermo (2) 31, 267-288 (1982) Zbl 0497.35078 MR 0670401

[12] Dias, J. P., Figueira, M.: On the existence of a global solution of the Cauchy problem for a Klein-Gordon-Dirac system. J. Math. Pures Appl. (9) 70, 75-85 (1991) Zbl 0662.35073 MR 1091920

[13] Eardley, D. M., Moncrief, V.: The global existence of Yang-Mills-Higgs fields in 4dimensional Minkowski space, I, II. Comm. Math. Phys. 83, 171-191 and 193-212 (1982) Zb1 0496.35061, 0496.35062 MR 0649158, 0649159

[14] Fang, Y. F.: On the Dirac-Klein-Gordon equations in one space dimension. Differential Integral Equations 17, 1321-1346 (2004) MR 2100030

[15] Fang, Y. F., Grillakis, M.: On the Dirac-Klein-Gordon equations in three space dimensions. Comm. Partial Differential Equations 30, 783-812 (2005) Zbl 1083.35121 MR 2153515

[16] Foschi, D., Klainerman, S.: Bilinear space-time estimates for homogeneous wave equations. Ann. Sci. École Norm. Sup. (4) 23, 211-274 (2000) Zbl 0959.35107 MR 1755116

[17] Gibbeson, D.: Regularity results for nonlinear wave equations. PhD thesis, Edinburgh (2004)

[18] Glassey, R. T., Strauss, W. A.: Conservation laws for the classical Maxwell-Dirac and KleinGordon-Dirac equations. J. Math. Phys. 20, 454-458 (1979) MR 0525106

[19] Kenig, C., Ponce, G., Vega, L.: The Cauchy problem for the KdV equation in Sobolev spaces of negative indices. Duke Math. J. 71, 1-21 (1993) Zbl 0787.35090 MR 1230283 
[20] Klainerman, S.: Global existence of small amplitude solutions to nonlinear Klein-Gordon equations in four space-time dimensions. Comm. Pure Appl. Math. 38, 631-64 (1985) Zbl 0597.35100 MR 0803252

[21] Klainerman, S., Machedon, M.: On the regularity properties of the wave equation. In: Physics on Manifolds (Paris, 1992), Math. Phys. Stud. 15, Kluwer, Dordrecht, 177-191 (1994) Zbl 0833.35093 MR 1267075

[22] Klainerman, S., Machedon, M.: Space-time estimates for null forms and the local existence theorem. Comm. Pure Appl. Math. 46, 1221-1268 (1993) Zbl 0803.35095 MR 1231427

[23] Klainerman, S., Machedon, M.: On the Maxwell-Klein-Gordon equation with finite energy. Duke Math. J. 74, 19-44 (1994) Zbl 0818.35123 MR 1271462

[24] Klainerman, S., Machedon, M.: Finite energy solutions for the Yang-Mills equations in $\mathbb{R}^{3+1}$. Ann. of Math. 142, 39-119 (1995) Zbl 0827.53056 MR 1338675

[25] Klainerman, S., Machedon, M.: Smoothing estimates for null forms and applications. Duke Math. J. 81, 99-133 (1995) Zbl 0909.35094 MR 1381973

[26] Klainerman, S., Machedon, M.: Remark on Strichartz type inequalities. Int. Math. Res. Not. 1996, no. 5, 201-220 Zbl 0853.35062 MR 1383755

[27] Klainerman, S., Selberg, S.: Bilinear estimates and applications to nonlinear wave equations. Commun. Contemp. Math. 4, 223-295 (2002) Zbl pre01782982 MR 1901147

[28] Lindblad, H.: Counterexamples to local existence for semilinear wave equations. Amer. J. Math. 118, 1-16 (1996) Zbl 0855.35080 MR 1375301

[29] Machedon, M., Sterbenz, J.: Almost optimal local well-posedness for the (3+1)-dimensional Maxwell-Klein-Gordon equations. J. Amer. Math. Soc. 17, 297-359 (2004) Zbl 1048.35115 MR 2051613

[30] Machihara, S.: The Cauchy problem for the 1-D Dirac-Klein-Gordon equation. Preprint (2006)

[31] Pecher, H.: Low regularity well-posedness for the one-dimensional Dirac-Klein-Gordon system. Electron. J. Differential Equations 2006, no. 150, 13 pp. Zbl 1115.35109 MR 2276575

[32] Ponce, G., Sideris, T.: Local regularity of nonlinear wave equations in three space dimensions. Comm. Partial Differential Equations 18, 169-177 (1993) Zbl 0803.35096 MR 1211729

[33] Selberg, S.: Multilinear spacetime estimates and applications to local existence theory for nonlinear wave equations. Ph.D. thesis, Princeton Univ. (1999)

[34] Selberg, S.: On an estimate for the wave equation and applications to nonlinear problems. Differential Integral Equations 2, 213-236 (2002) Zbl 1032.35121 MR 1870470

[35] Strichartz, R. S.: Restriction of Fourier transforms to quadratic surfaces and decay of solutions of wave equations. Duke Math. J. 44, 705-714 (1977) Zbl 0372.35001 MR 0512086

[36] Tao, T.: Multilinear weighted convolution of $L^{2}$ functions, and applications to nonlinear dispersive equations. Amer. J. Math. 123, 839-908 (2001) Zbl 0998.42005 MR 1854113 